\title{
Combination leflunomide and methotrexate impedes the recovery of liver fibrosis, partly through inhibition of myeloid cell admittance
}

\author{
MENGXIN LIN, RUYI GUO, ZHIJUN SU, SHAOPENG KE and DAWU ZENG \\ Department of Infectious Disease, The First Hospital of Quanzhou City, Quanzhou, Fujian 362000, P.R. China
}

Received March 19, 2018; Accepted October 25, 2018

DOI: $10.3892 / \mathrm{mmr} .2019 .9821$

\begin{abstract}
The process of liver fibrosis is reversible and involves a recovery phase. In the present study, the potential side effects of combination leflunomide and methotrexate (LEF+MTX), a conventional rheumatoid arthritis therapy used in the resolution of liver fibrosis, was investigated. In a carbon tetrachloride-induced liver fibrosis model, the results of hepatic pathology demonstrated that the LEF+MTX combination delayed the recovery of fibrosis, although the activation of hepatic stellate cells in vitro was inhibited. A total of four liver fibrosis-associated indicators, hyaluronic acid, laminin, procollagen type III and collagen IV, maintained high levels in the serum of LEF+MTX-treated mice, while detection of bone marrow-driven monocytes in the blood by flow cytometry indicated that they were significantly decreased. Notably, the results of immunofluorescence staining of hepatic myeloid cells and detection of vascular growth factor A (VEGF-A) in blood and liver suggested that the reduced degeneration of collagen in liver sinusoids was associated with decreased myeloid cell adhesion and the downregulation of VEGF-A in the liver. The present results suggested that in certain cases, treatment with LEF+MTX may impede the recovery of hepatic fibrosis-associated diseases in mice.
\end{abstract}

\section{Introduction}

Chronic liver disease, the leading cause of mortality and morbidity globally, is characterized as fibrogenesis, which increases the risk of liver cirrhosis and liver failure (1). Persistent liver injury leads to unresolved inflammation and the activation of myofibroblasts, which express extracellular matrix (ECM) components to trigger the generation of liver fibrosis (2). However, observations in clinical studies and experimental

Correspondence to: Dr Mengxin Lin, Department of Infectious Disease, The First Hospital of Quanzhou City, 250 East Street, Quanzhou, Fujian 362000, P.R. China

E-mail: mengxinlin1980@163.com

Key words: leflunomide, methotrexate, liver fibrosis, myeloid cell, vascular growth factor animal models suggest that the process of liver fibrosis is dynamic and reversible, indicating that it contains at least two phases, the developmental and recovery phases (3). Therefore, the guidance of clinical medication may need to be upgraded according to the different phases of this disease, which might have an influence on the side-effects of treatments.

Leflunomide (LEF) is an anti-rheumatic drug with immunosuppressive and anti-inflammatory functions (4). Teriflunomide, the metabolite of LEF, induces cell cycle arrest in vigorously dividing lymphocytes, and not in non-lymphatic cells (5). Methotrexate (MTX) is a chemotherapy agent used for autoimmune diseases due to its inhibitory effect on lymphocyte activation (6). Of note, following failure of monotherapy, LEF is used in combination with MTX, which has been demonstrated to be effective and safe (6). However, clinical studies suggest that this combination is associated with a significantly increased risk of leucopenia and silent liver fibrosis in patients with rheumatoid arthritis (RA) (7,8). Chronic liver diseases frequently overlap with patients suffering from rheumatoid disease, which requires the disease-modifying anti-rheumatic drugs to withstand an excessive autoimmune response (9). Thus, studying the multiple effects of the anti-rheumatic drugs in the process of liver fibrosis is of importance. Additionally, previous studies highlighted that the resolution of liver fibrosis is mediated by myeloid cell-induced sinusoidal remodeling and the involvement of macrophages $(10,11)$. Therefore, the potential side effects of the LEF+MTX combination during the spontaneous recovery phase of liver fibrosis in mice was investigated.

In the present study, it was demonstrated that the LEF+MTX treatment impeded the step of hepatic fibrosis recovery. The underlying mechanism was associated with hindering ECM degradation and sinusoidal remodeling induced by the reduced adhesion of myeloid cells. The present study elucidated the side effects of the LEF+MTX combination in hepatic fibrosis recovery and provided evidence for its use in clinical therapy.

\section{Materials and methods}

Animal experiment. A total of 50, 6-week old C57BL/6 mice weighing $20 \mathrm{~g}$ were obtained from the Shanghai Model Organisms Center, Inc. (Shanghai, China). Mice were divided into 4 groups, 6 mice per group, for 2 individual experiments. All animal experiments were approved by the Committee on Laboratory Animal Care of Fujian Medical University 
(Fuzhou, China) and performed according to the 'ARRIV' guidelines (12). Female mice aged 8 weeks were intraperitoneally injected with $200 \mu \mathrm{l} 25 \%$ carbon tetrachloride $\left(\mathrm{CCl}_{4}\right)$ /olive oil $(2 \mathrm{ml} / \mathrm{kg})$ three times per week for 12 weeks to induce hepatic fibrosis. A group of mice served as the control, termed the NC group. The degree of liver fibrosis in the mice was evaluated by histology. Subsequently, the mice were randomly divided into two groups: A group received LEF $(15 \mathrm{mg} / \mathrm{kg}$, oral administration) and MTX (intraperitoneal administration of $0.15 \mathrm{mg} / \mathrm{Kg}$,) every day for four weeks and a group that received the relevant vehicle of LEF and MTX via the same routes. Leflunomide (solvent, $0.5 \%$ sodium carboxymethylcellulose) and methotrexate (solvent, PBS) were purchased from Sigma-Aldrich (Merck KGaA, Darmstadt, Germany).

Hematoxylin-eosin staining. Whole liver tissues were fixed in $4 \%$ paraformaldehyde at room temperature overnight and embedded in paraffin. Sections $(5 \mu \mathrm{m})$ of paraffin-embedded tissues were subjected to hematoxylin staining for $8 \mathrm{~min}$ at room temperature. Following washing in water for $1 \mathrm{~h}$ and dehydration in ethyl alcohol for $10 \mathrm{~min}$, the sections were subjected to eosin staining for $3 \mathrm{~min}$ at room temperature. Following dehydration in ethyl alcohol, the sections were mounted to detect pathological alterations. The images (magnification, x200) were obtained by an Axio Vert.A1 inverted microscope (Zeiss AG, Oberkochen, Germany).

Masson staining. Masson staining was performed with a Masson-Goldner staining kit (Merck KGaA), following the manufacturer's protocol, to detect collagen deposition in liver tissues. Briefly, rehydrated sections $(5 \mu \mathrm{m})$ were staining with Weigert's iron hematoxylin staining solution for $5 \mathrm{~min}$, followed by staining with Azophloxine solution for $10 \mathrm{~min}$, Tungstophosphoric acid orange G solution for 1 min and Light green SF solution for $2 \mathrm{~min}$. Washes with $1 \%$ acetic acid were performed between the staining steps. Following dehydration in ethyl alcohol, the sections were mounted. All the procedures were performed on room temperature. The images (magnification, x200) were obtained by an Axio Vert.A1 inverted microscope (Zeiss AG, Oberkochen, Germany).

Immunofluorescence. Liver tissues were fixed in $4 \%$ paraformaldehyde and cut using a cryostat. Sections $(10 \mu \mathrm{m})$ were permeabilized with PBS containing $0.5 \%$ Triton X-100. Following blocking with PBS/5\% bovine serum (Gibco; Thermo Fisher Scientific, Inc., Waltham, MA, USA) for $2 \mathrm{~h}$ at room temperature, the sections were incubated with Alexa-Fluor488-conjugated rat anti-mouse cluster of differentiation (CD)11b antibody (1:200; cat. no. 101219; clone M1/70; BioLegend, Inc., San Diego, CA, USA) at $37^{\circ} \mathrm{C}$ for $1 \mathrm{~h}$ in the dark. The sections were counterstained with DAPI (BioLegend, Inc.) for $15 \mathrm{~min}$ at room temperature. The images (magnification, x200) were visualized by a Zeiss LSM700 confocal microscope (Zeiss AG).

ELISA. Serum $(\sim 5 \mu \mathrm{l})$ contents were detected by procollagen type III (cat. no. CSB-E17125m), collagen type IV (cat. no. CSB-E08884m), hyaluronic acid (cat. no. CSB-E08121m) and laminin (cat. no. CSB-EL012725MO) ELISA kits (all Cusabio, Wuhan, China), according to the manufacturer's protocols. To detect the expression levels of liver vascular endothelial growth factor (VEGF) protein, liver tissues were homogenized in radioimmunoprecipitation (RIPA; Cusabio Technology LLC, Wuhan, China) buffer on ice, and the supernatants of the tissue homogenate were measured using a VEGF ELISA kit (cat. no. CSB-E04756m; Cusabio, Wuhan, China).

Flow cytometry. Whole blood samples (100 $\mu \mathrm{l})$ were incubated with red blood cell lysis buffer (BD Pharmingen; BD Biosciences, San Jose, CA, USA). Following centrifugation at $500 \mathrm{x}$ g for $15 \mathrm{~min}$ at $4^{\circ} \mathrm{C}$, the cells were washed, stained with purified anti-CD16/32 (1/200; clone 2.4G2; cat. no. 553141; BD Pharmingen; BD Biosciences), and subsequently stained with fluorescein isothiocyanate-conjugated CD11b (1:400; clone M1/70; cat. no. 557396; BD Pharmingen; BD Biosciences) and allophycocyanin-conjugated Ly6C (1:20; clone HK1.4; cat. no. 560595; BD Pharmingen; BD Biosciences) antibodies for $30 \mathrm{~min}$ at $4^{\circ} \mathrm{C}$. The cells were washed, resuspended in $200 \mu \mathrm{l}$ FACS buffer (BD Pharmingen; BD Biosciences) and analyzed with a FACSAria (BD Biosciences). FlowJo software (version 10; FlowJo, LLC, Ashland, OR, USA) was used for analysis. The number $(\mathrm{N})$ cells per $\mathrm{ml}$ in blood was calculated by the formula: $\mathrm{N}=$ collected cells $\mathrm{x}$ 200/loading volume $\mathrm{x} 10$.

Reverse transcription-quantitative polymerase chain reaction (RT-qPCR) in liver tissues. Sections of liver tissues (4 mm) were rapidly frozen in liquid nitrogen and homogenized in $1 \mathrm{ml} \mathrm{TRIzol}{ }^{\circledR}$ reagent (Thermo Fisher Scientific, Inc.). The RNA was exacted following the manufacturer's protocol, and cDNA was synthesized using SuperScriptIIreverse transcriptase (Invitrogen; Thermo Fisher Scientific, Inc.) and oligodT primers (Invitrogen; Thermo Fisher Scientific, Inc.) according to the manufacturer's protocol. The primers were as follows: VEGF-A forward, 5'-ATCCGCATGATCTGCATGG-3' and reverse, 5'-ATCCGCATGATCTGCATGG-3'; beta-actin forward, 5'-CCACCATGTACCCAGGCATT-3' and reverse, 5'-CGGACTCATCGTACTCCTGC-3'. RT-qPCR was performed with cDNA in $15 \mu$ l reactions using SYBR-Green Master Mix (Promega Corporation, Madison, WI, USA). The thermocycling conditions were: $94{ }^{\circ} \mathrm{C}$ for $10 \mathrm{~min}, 94^{\circ} \mathrm{C}$ for $30 \mathrm{sec}, 60^{\circ} \mathrm{C}$ for $30 \mathrm{sec}$ and $72^{\circ} \mathrm{C}$ for $30 \mathrm{sec}$, repeated for 35 cycles. The mRNA levels were first calculated using the $2^{-\Delta \Delta \mathrm{Cq}}$ method and then normalized to $\beta$-actin level (13).

Statistical analysis. All graphs were manufactured using GraphPad Prism version 7.0 software (GraphPad Software, Inc., La Jolla, CA, USA). Statistical analysis was analyzed by one-way analysis of variance followed by Tukey's multiple comparisons test. Data are expressed as the mean \pm standard deviation and collected from at least 6 mice. $\mathrm{P}<0.05$ was considered to indicate a statistically significant difference.

\section{Results}

Combination LEF+MTX impedes the recovery of liver pathology in the liver fibrosis in mice. The animal experiments were performed as illustrated in Fig. 1A. After 12 weeks of $\mathrm{CCl}_{4}$ administration, five mice were randomly selected for the determination of liver pathology, and illustrating a marked alteration in the liver histology (Fig. 1B). Subsequently, the mice with liver fibrosis were divided into two groups with 


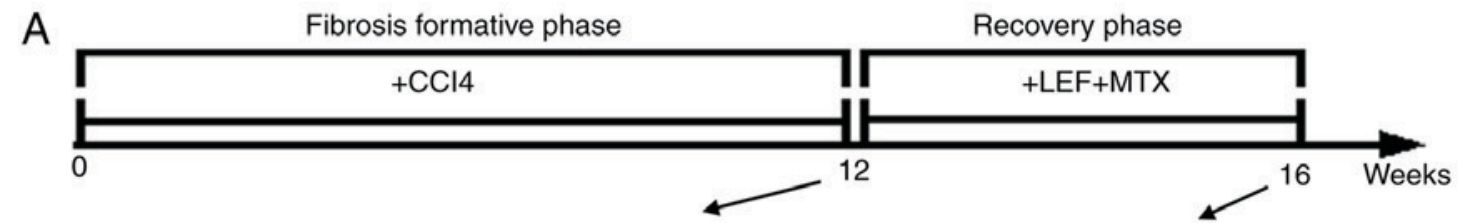

B
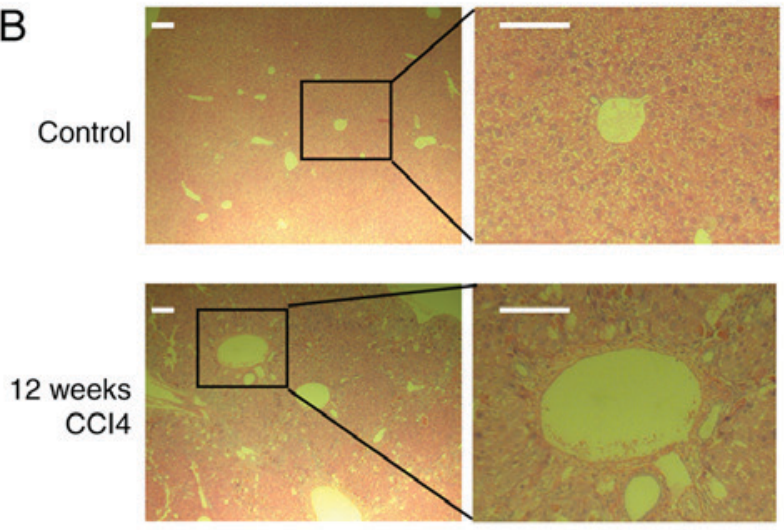

C
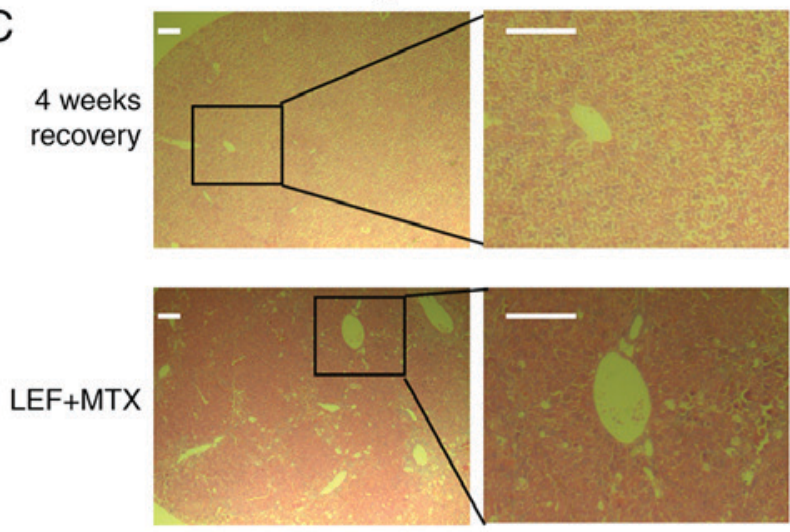

Figure 1. Mice with liver fibrosis receiving the LEF+MTX combination exhibit severe liver injury after 4 weeks of recovery. (A) Schematic representation of the animal experiments. (B) Representative images from the control and model mice $(\mathrm{n}=5)$. (C) Representative images from mice treated with $\mathrm{LEF}+\mathrm{MTX}$ and mice that experienced spontaneous recovery $(\mathrm{n}=6)$. Scale bar, $200 \mu \mathrm{m} . \mathrm{CCl}_{4}$, carbon tetrachloride; LEF, leflunomide; MTX, methotrexate.

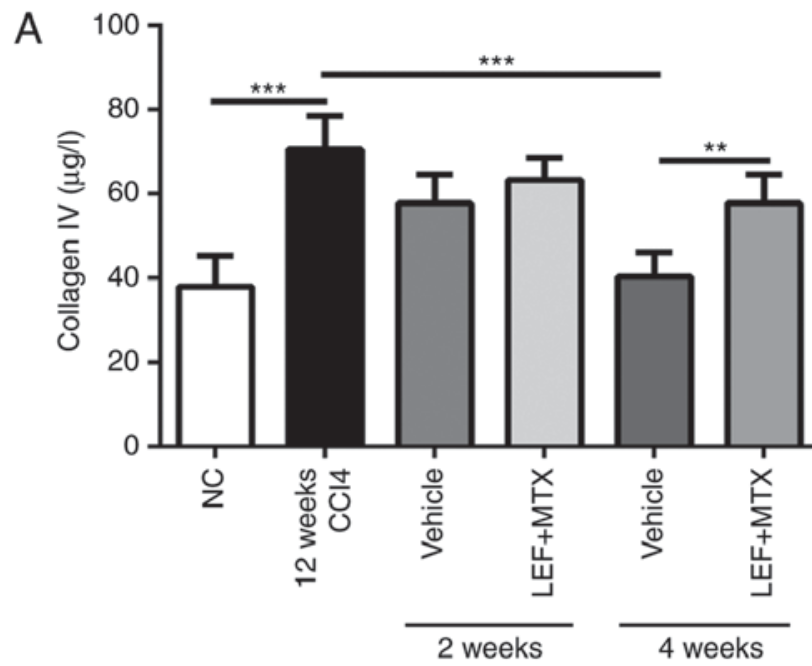

C

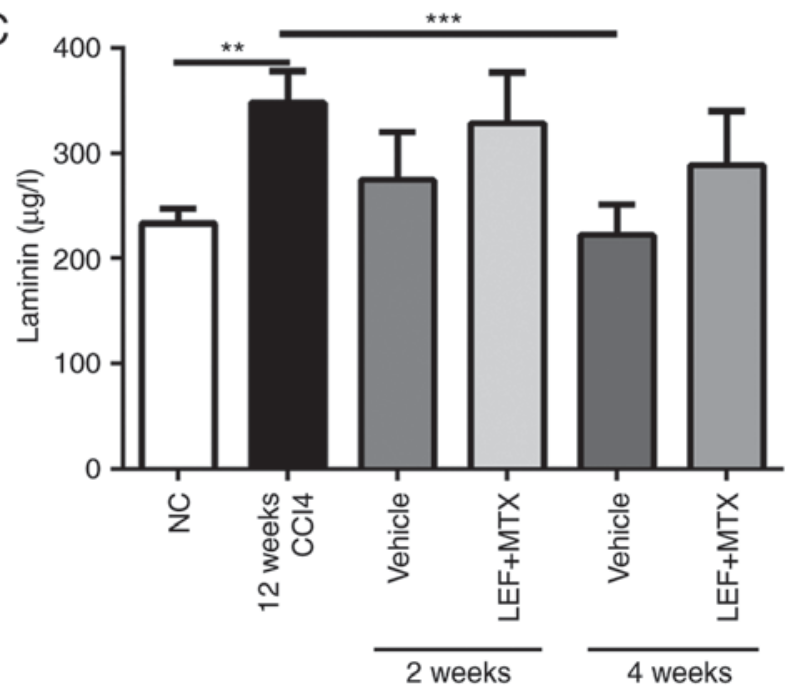

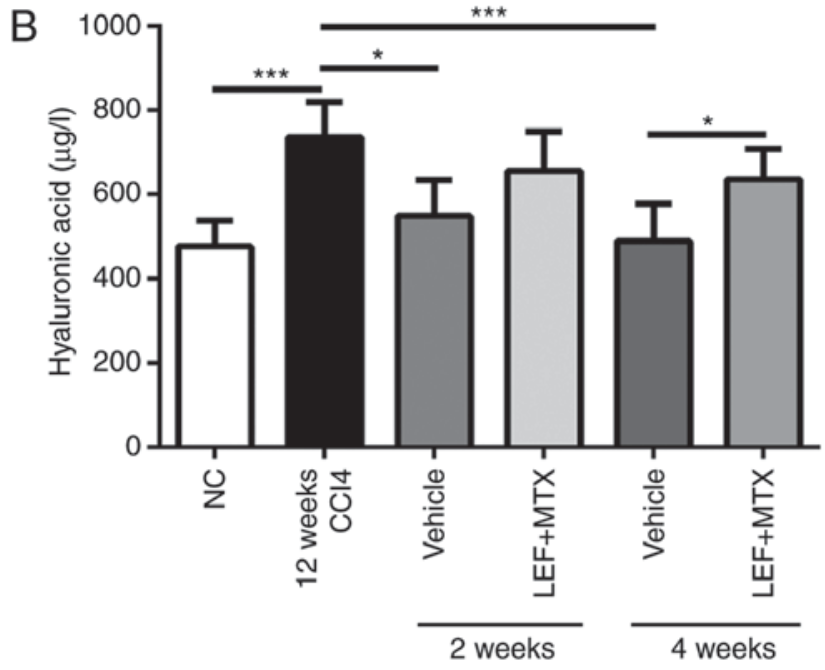

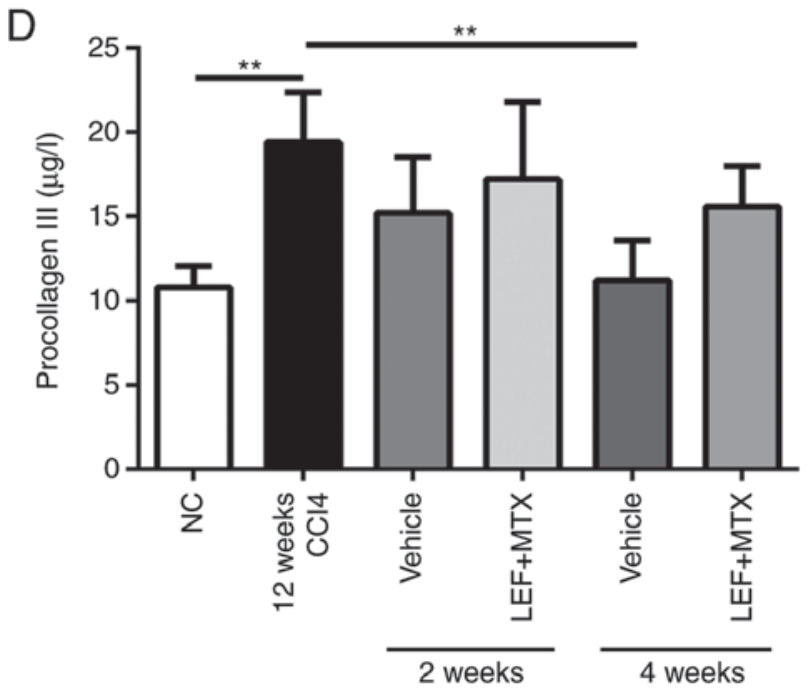

Figure 2. Serum collagen is sustained at high levels in mice treated with LEF+MTX. The serum was collected at weeks 2 and 4 during the recovery phase. The levels of (A) collagen IV, (B) hyaluronic acid, (C) laminin and (D) procollagen III in serum were measured by ELISA. Data are presented as the mean \pm standard deviation $(\mathrm{n}=6) .{ }^{*} \mathrm{P}<0.05,{ }^{* *} \mathrm{P}<0.01$ and ${ }^{* * *} \mathrm{P}<0.001 . \mathrm{CCl}_{4}$, carbon tetrachloride; LEF, leflunomide; MTX, methotrexate; NC, normal control. 

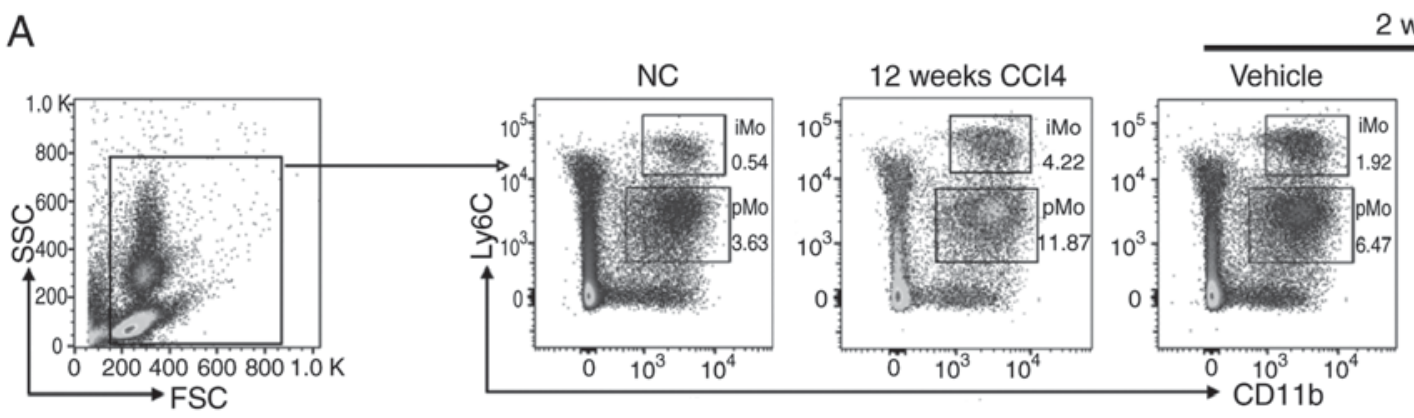

2 weeks
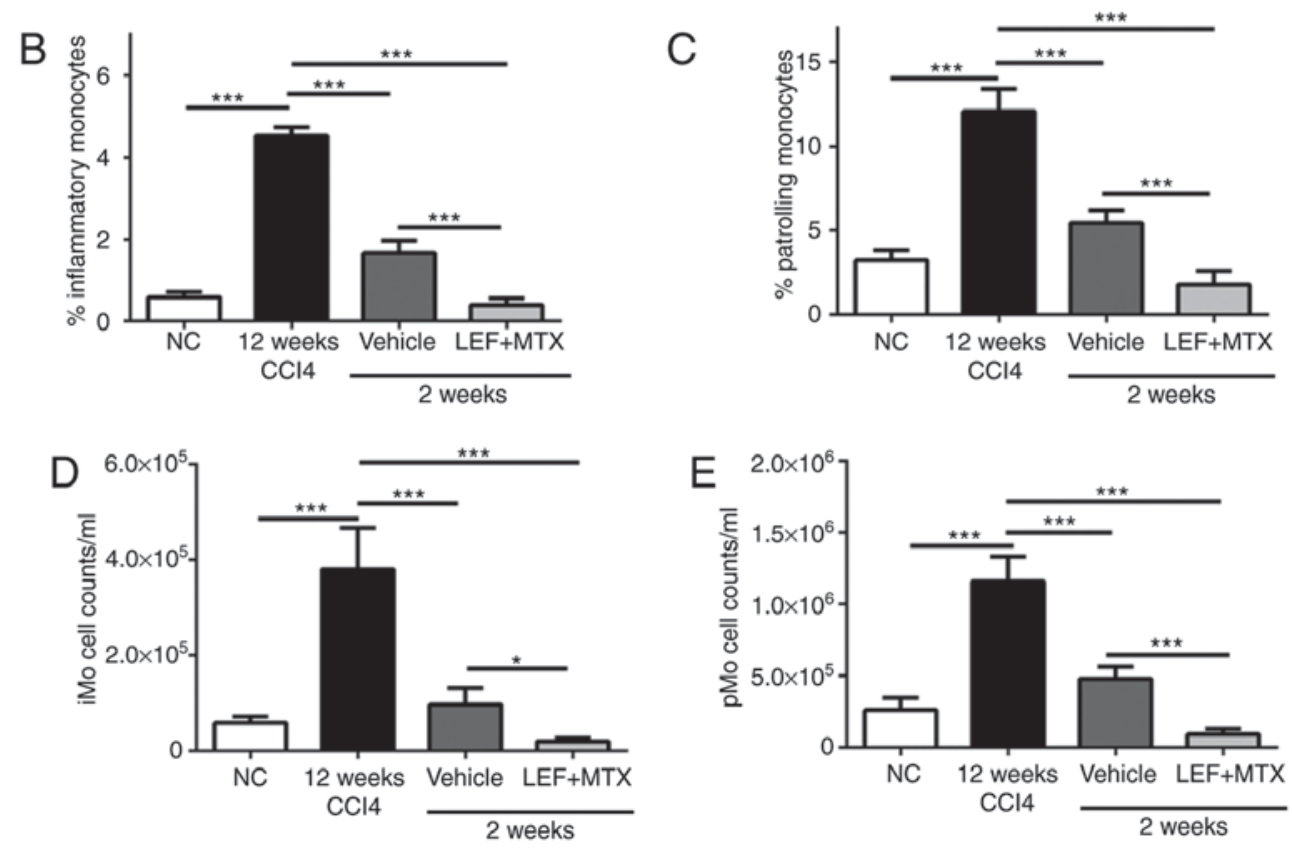

Figure 3. Monocyte subsets are significantly decreased following treatment with LEF+MTX. Whole blood (200 $\mu \mathrm{l})$ was collected, and the red blood cells were removed. (A) The gating strategy and representative images of flow cytometry for CD11b ${ }^{+} \mathrm{Ly} 6 \mathrm{C}^{+}$cells. The percentages of (B) iMo (CD11b $\left.{ }^{+} \mathrm{Ly} 6 \mathrm{Chigh}\right)$ and of $(\mathrm{C}) \mathrm{pMo}\left(\mathrm{CD} 11 \mathrm{~b}^{+} \mathrm{Ly} 6 \mathrm{Cint}\right)$ were measured by flow cytometry. The absolute numbers of (D) iMo and (E) pMo are also presented. Data are presented as the mean \pm standard deviation $(\mathrm{n}=6) .{ }^{*} \mathrm{P}<0.05$ and ${ }^{* * *} \mathrm{P}<0.001 . \mathrm{CCl}_{4}$, carbon tetrachloride; $\mathrm{CD}$, cluster of differentiation; iMo, inflammatory monocytes; LEF, leflunomide; MTX, methotrexate; NC, normal control; pMo, patrolling monocytes; FSC, forward scatter; SSC, side scatter.

or without treatment with the LEF+MTX combination, and underwent recovery for 4 weeks. A completely spontaneous recovery was found in the mice without further intervention (Fig. 1C, upper panel). By contrast, the mice that received the LEF+MTX combination exhibited severe liver pathology compared with the control group (Fig. 1C, lower panel). These results demonstrated that the LEF+MTX combination had an inhibitory effect on the recovery of liver fibrosis.

A high level of collagen degradation is observed in mice receiving $L E F+M T X$ treatment. Liver fibrosis levels are accompanied by increasing levels of serum indicators, including hyaluronic acid, laminin, procollagen type III and collagen IV (14). The mice which underwent 12 weeks of $\mathrm{CCl}_{4}$ administration demonstrated a marked increase in serum parameters. Following grouping, the mice without LEF+MTX treatment exhibited a continuous decline of these indicators, which almost returned to their normal levels after 4 weeks (Fig. 2). By contrast, no significant decrease of these indicators was observed in the mice that received the LEF+MTX combination. In addition, compared with mice without LEF+MTX treatment, mice that were administrated with LEF+MTX combination showed the higher levels of collagen IV and hyaluronic acid.

Reduced monocytes in blood are associated with the impaired resolution of fibrosis. It has been demonstrated that combination LEF+MTX has a potent effect on the inhibition of leucopoiesis (7). The absolute numbers and percentages of monocytes in blood from the mice that experienced recovery at 2 weeks were measured. The gating strategy used to identify the inflammatory monocytes (iMo, CD11b+Ly6 $\mathrm{C}^{\text {high }}$ ) and the patrolling monocytes (pMo, CD11b+Ly6C int) is illustrated in Fig. 3A. Treating mice with $\mathrm{CCl}_{4}$ for 12 weeks significantly increased the number of monocytes in the blood. After 2 weeks of recovery, iMo and pMo were decreased in the mice, whether or not they were treated with LEF+MTX (Fig. 3B-E). Compared with the vehicle group, either the absolute numbers or cell percentages of iMo and pMo were decreased in the LEF+MTX-treated mice. In summary, the LEF+MTX combination significantly reduced the levels of two monocyte subsets in the mice that underwent fibrosis recovery, and this may cause the impaired recruitment of monocytes to the site of liver fibrosis, although this requires further investigation. 
A
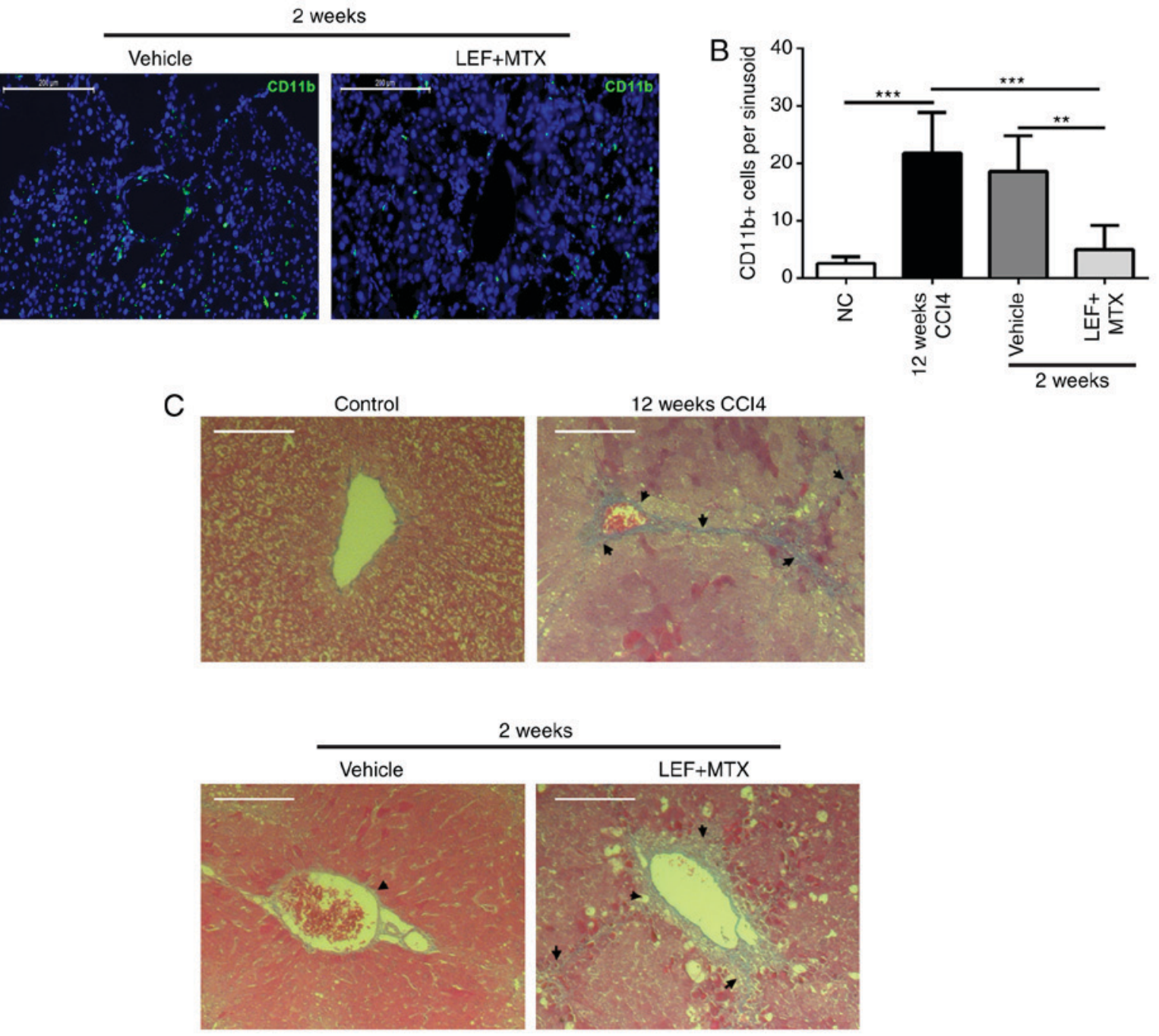

weeks
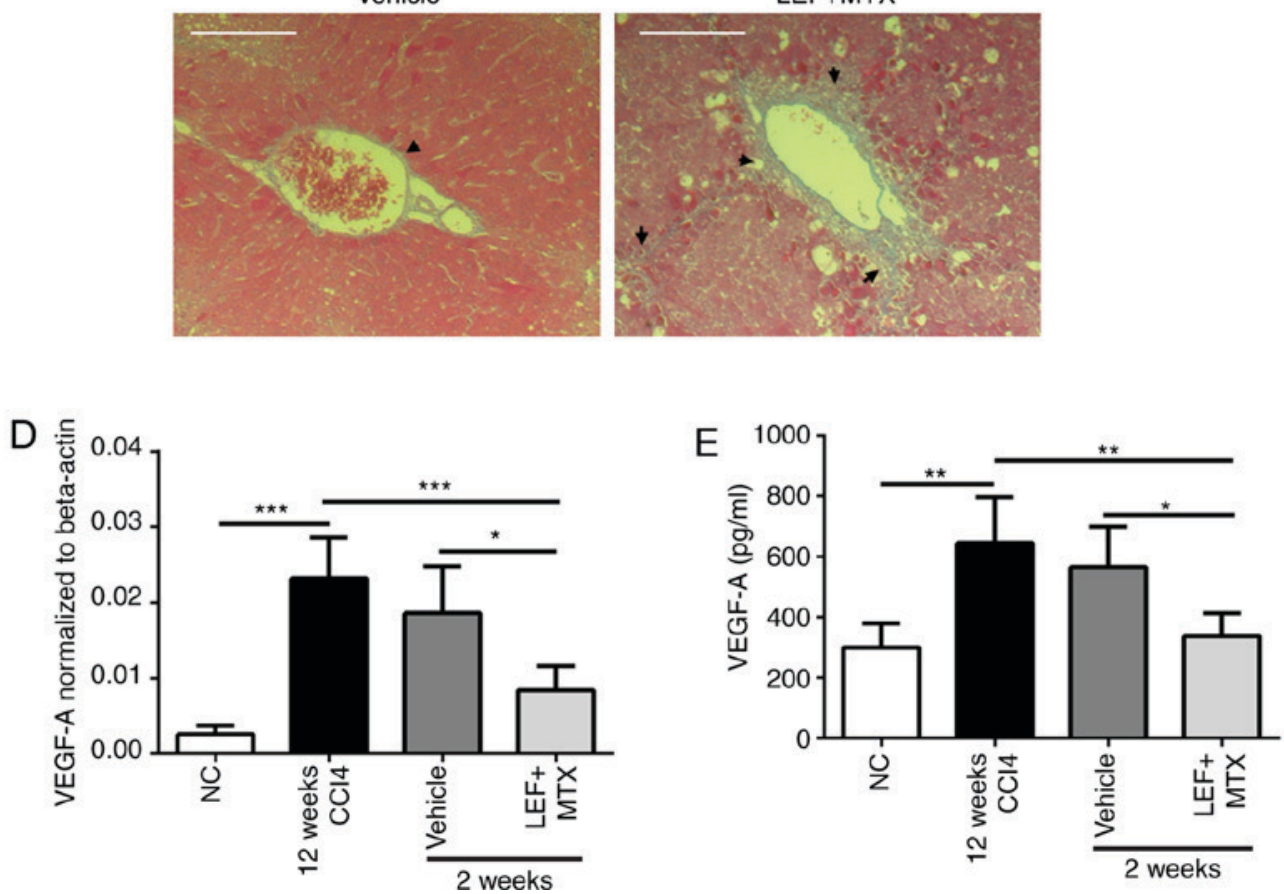

Figure 4. Combination LEF+MTX suppresses myeloid cell adhesion to the sinusoidal endothelium and hinders fibrosis resolution. (A) Representative images of the liver sinusoidal endothelium illustrating the $\mathrm{CD} 11 \mathrm{~b}^{+}$cells (green). (B) $\mathrm{CD} 11 \mathrm{~b}^{+}$cells per liver sinusoid were calculated. (C) Representative images of Masson staining. (D) mRNA and (E) protein expression levels of VEGF-A were detected by reverse transcription-quantitative polymerase chain reaction and ELISA,

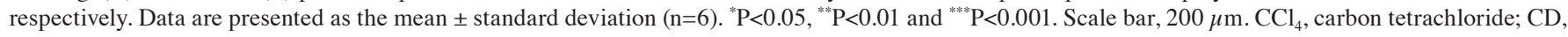
cluster of differentiation; LEF, leflunomide; MTX, methotrexate; NC, normal control; VEGF, vascular endothelial growth factor.

Combination LEF+MTX suppresses fibrosis resolution mediated by myeloid cells during the recovery phase. To reveal the underlying association between myeloid cells in the blood and fibrosis resolution, CD11b immunohistochemistry and Masson's trichrome staining were performed on the liver tissues. The results demonstrated that during the recovery phase, abundant $\mathrm{CD}_{11} \mathrm{~b}^{+}$cells were adhered to the sinusoidal endothelium, while the LEF+MTX treatment significantly reduced the

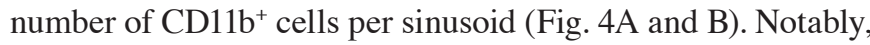
the results of the Masson's trichrome staining suggested a marked degradation of the ECM in liver sinusoids from mice that experienced the spontaneous recovery, although not in the mice treated with the LEF+MTX combination (Fig. 4C). Subsequently, the expression levels of VEGF-A, a cytokine 
involved in angiogenesis which is also present in this phase, were investigated (10). The mRNA and protein expression levels of VEGF-A demonstrated that compared with the mice that had undergone recovery at 2 weeks, the VEGF-A expression levels in the LEF+MTX-treated mice were decreased (Fig. 4D and E). These results indicated that the LEF+MTX combination may suppress the presence of myeloid cells in the liver sinusoidal endothelium, impeding the fibrosis recovery.

\section{Discussion}

Hepatic fibrosis, a pathological consequence of chronic liver diseases with differing etiologies, is characterized by the deposition of ECM produced by activated hepatic stellate cells in response to persistent inflammation and unremitting injury (2). A previous clinical study demonstrated that treatment with combined LEF and MTX in patients with RA led to an increased risk of silent liver fibrosis (8). In the present study, part of the underlying mechanism of this was assessed in experimental animals.

Previous studies have proposed that LEF exerts an inhibitory effect on the formation of hepatic fibrosis, accelerating the fibrosis recovery that involves the enhanced apoptosis of hepatic stellate cells $(15,16)$. Additionally, MTX is associated with the increased morbidity of hepatic fibrosis and increased myelosuppression, which is characterized by fewer white blood cells in the blood, particularly myeloid cell subsets $(17,18)$. In the recovery phase of liver fibrosis, it was observed that the LEF+MTX combination significantly impeded the resolution of the fibrotic scar, and the underlying mechanism may be associated with impaired myeloid cell-induced remodeling of the liver sinusoids. Inflammatory monocytes selectively respond to infection and tissue damage, and are also able to digest damaged tissue $(19,20)$. pMo, with attenuated inflammatory properties, exhibit a fundamental characteristic of patrolling the endothelium and, notably, they are the principal producers of VEGF-A and drive healing via the accumulation of myofibroblasts, angiogenesis and the deposition of ECM (20). These characteristics of monocyte subsets support the observation that the LEF+MTX combination inhibited the number of monocytes in circulation, leading to fewer monocytes reaching the site of liver fibrosis recovery. A previous study on the recovery of liver fibrosis suggested that myeloid cell-driven VEGF is indispensable for the degradation of the ECM in liver sinusoids, and that this process is dependent on the regulation of sinusoidal cells (10). This notion is consistent with the present data, in that decreased expression of liver VEGF-A was associated with impaired ECM degradation. In addition, a previous in vivo study suggested that LEF administration increases hepatic exposure to MTX, which may lead to additional liver injury (21). This observation may be another reason why the LEF+MTX administration impeded fibrosis recovery.

In the present study, it was demonstrated that during the recovery phase of $\mathrm{CCl}_{4}$-induced liver fibrosis, the LEF+MTX treatment impeded fibrosis resolution by reducing the number of myeloid cells, which may promote the revascularization of sinusoidal endothelial cells, leading to reduced ECM degradation. The present study highlighted a potential side effect of the LEF+MTX combination in the context of recovery from hepatic fibrosis-associated diseases.

\section{Acknowledgements}

Not applicable.

\section{Funding}

No funding was received.

\section{Availability of data and materials}

All data generated or analyzed during this study are included in this published article.

\section{Authors' contributions}

ML, RG and ZS performed the animal experiments and analyzed data. ML, SK and DZ contributed to the conception and design of this study. ML drafted this manuscript. All authors have reviewed this manuscript and approved for publication.

\section{Ethics approval and consent to participate}

All animal experiments were approved by the Committee on Laboratory Animal Care of Fujian Medical University (Fuzhou, China) and performed according to the 'ARRIV' guidelines.

\section{Patient consent for publication}

Not applicable.

\section{Competing interests}

The authors declare that they have no competing interests

\section{References}

1. Patten DA and Shetty S: Chronic liver disease: Scavenger hunt for novel therapies. Lancet 391: 104-105, 2018.

2. Tsuchida T and Friedman SL: Mechanisms of hepatic stellate cell activation. Nat Rev Gastroenterol Hepatol 14: 397-411, 2017.

3. Pellicoro A, Ramachandran P, Iredale JP and Fallowfield JA Liver fibrosis and repair: Immune regulation of wound healing in a solid organ. Nat Rev Immunol 14: 181-194, 2014.

4. Janssen NM and Genta MS: The effects of immunosuppressive and anti-inflammatory medications on fertility, pregnancy, and lactation. Arch Intern Med 160: 610-619, 2000.

5. Dougados M, Emery P, Lemmel EM, Zerbini CA, Brin S and van Riel P: When a DMARD fails, should patients switch to sulfasalazine or add sulfasalazine to continuing leflunomide? Ann Rheum Dis 64: 44-51, 2005

6. Wessels JA, Huizinga TW and Guchelaar HJ: Recent insights in the pharmacological actions of methotrexate in the treatment of rheumatoid arthritis. Rheumatology (Oxford) 47: 249-255, 2008.

7. Rozman B: Clinical pharmacokinetics of leflunomide. Clin Pharmacokinet 41: 421-430, 2002.

8. Lee SW, Park HJ, Kim BK, Han KH, Lee SK, Kim SU and Park YB: Leflunomide increases the risk of silent liver fibrosis in patients with rheumatoid arthritis receiving methotrexate. Arthritis Res Ther 14: R232, 2012.

9. Ferri C, Sebastiani M, Antonelli A, Colaci M, Manfredi A and Giuggioli D: Current treatment of hepatitis C-associated rheumatic diseases. Arthritis Res Ther 14: 215, 2012. 
10. Kantari-Mimoun C, Castells M, Klose R, Meinecke AK, Lemberger UJ, Rautou PE, Pinot-Roussel H, Badoual C, Schrödter K, Österreicher $\mathrm{CH}$, et al: Resolution of liver fibrosis requires myeloid cell-driven sinusoidal angiogenesis. Hepatology 61: 2042-2055, 2015.

11. Duffield JS, Forbes SJ, Constandinou CM, Clay S, Partolina M, Vuthoori S, Wu S, Lang R and Iredale JP: Selective depletion of macrophages reveals distinct, opposing roles during liver injury and repair. J Clin Invest 115: 56-65, 2005.

12. Kilkenny C, Browne W, Cuthill IC, Emerson M, Altman DG and National Centre for the Replacement, Refinement and Reduction of Amimals in Research: Animal research: Reporting in vivo experiments-the ARRIVE guidelines. J Cereb Blood Flow Metab 31: 991-993, 2011.

13. Livak KJ and Schmittgen TD: Analysis of relative gene expression data using real-time quantitative PCR and the 2(-Delta Delta C(T)) method. Methods 25: 402-408, 2001.

14. Fontana RJ, Dienstag JL, Bonkovsky HL, Sterling RK, Naishadham D, Goodman ZD, Lok AS, Wright EC and Su GL; HALT-C Trial Group: Serum fibrosis markers are associated with liver disease progression in non-responder patients with chronic hepatitis C. Gut 59: 1401-1409, 2010.

15. Yao HW, Li J, Chen JQ and Xu SY: Inhibitory effect of leflunomide on hepatic fibrosis induced by $\mathrm{CCl} 4$ in rats. Acta Pharmacol Sin 25: 915-920, 2004.

16. Tang X, Yang J and Li J: Accelerative effect of leflunomide on recovery from hepatic fibrosis involves TRAIL-mediated hepatic stellate cell apoptosis. Life Sci 84: 552-557, 2009.
17. Bjorkman DJ, Boschert M, Tolman KG, Clegg DO and Ward JR: The effect of long-term methotrexate therapy on hepatic fibrosis in rheumatoid arthritis. Arthritis Rheum 36: 1697-1701, 1993.

18. Bilasy SE, Essawy SS, Mandour MF, Ali EA and Zaitone SA: Myelosuppressive and hepatotoxic potential of leflunomide and methotrexate combination in a rat model of rheumatoid arthritis. Pharmacol Rep 67: 102-114, 2015.

19. Landsman L, Varol C and Jung S: Distinct differentiation potential of blood monocyte subsets in the lung. J Immunol 178: 2000-2007, 2007.

20. Nahrendorf M, Swirski FK, Aikawa E, Stangenberg L, Wurdinger T, Figueiredo JL, Libby P, Weissleder R and Pittet MJ: The healing myocardium sequentially mobilizes two monocyte subsets with divergent and complementary functions. J Exp Med 204: 3037-3047, 2007.

21. Wang L, Ma L, Lin Y, Liu X, Xiao L, Zhang Y, Xu Y, Zhou H and Pan G: Leflunomide increases hepatic exposure to methotrexate and its metabolite by differentially regulating multidrug resistance-associated protein Mrp2/3/4 transporters via peroxisome proliferator-activated receptor $\alpha$ activation. Mol Pharmacol 93: 563-574, 2018.

This work is licensed under a Creative Commons Attribution-NonCommercial-NoDerivatives 4.0 International (CC BY-NC-ND 4.0) License. 